\title{
Ontology Approximation in Horn Description Logics
}

\author{
Anneke Bötcher ${ }^{1}$, Carsten Lutz ${ }^{1}$, Frank Wolter ${ }^{2}$ \\ ${ }^{1}$ University of Bremen, Germany \\ ${ }^{2}$ University of Liverpool, UK \\ \{anneke, clu\}@uni-bremen.de, wolter@liverpool.ac.uk
}

\begin{abstract}
We study the approximation of a description logic (DL) ontology in a less expressive DL, focussing on the case of Horn DLs. It is common to construct such approximations in an ad hoc way in practice and the resulting incompleteness is typically neither analyzed nor understood. In this paper, we show how to construct complete approximations. These are typically infinite or of excessive size and thus cannot be used directly in applications, but our results provide an important theoretical foundation that enables informed decisions when constructing incomplete approximations in practice.
\end{abstract}

\section{Introduction}

There is a large number of description logics (DLs) that vary considerably regarding their expressive power and computational properties [Baader et al., 2017] and despite prominent standardization efforts, many different DLs continue to be used. ${ }^{1}$ As a result, it can be necessary to convert an ontology formulated in a DL $\mathcal{L}_{S}$, the source $D L$, into a different DL $\mathcal{L}_{T}$, the target DL. For example, this happens in ontology import when an engineer who designs an ontology formulated in $\mathcal{L}_{T}$ wants to reuse content from an existing ontology formulated in $\mathcal{L}_{S}$. A particularly important case is that $\mathcal{L}_{T}$ is a fragment of $\mathcal{L}_{S}$, in which case the described problem is ontology approximation, a form of knowledge compilation [Selman and Kautz, 1996; Darwiche and Marquis, 2002].

In this paper, we are interested in approximating an ontology $\mathcal{O}_{S}$ formulated in a DL $\mathcal{L}_{S}$ by an ontology $\mathcal{O}_{T}$ formulated in a fragment $\mathcal{L}_{T}$ of $\mathcal{L}_{S}$, aiming to preserve all information from $\mathcal{O}_{S}$ that is expressible in $\mathcal{L}_{T}$; this is called a greatest lower bound in knowledge compilation [Selman and Kautz, 1996]. Formally, for every $\mathcal{L}_{T}$ concept inclusion $C \sqsubseteq D$ that is formulated in the signature $\Sigma$ of $\mathcal{O}_{S}$, we require that $\mathcal{O}_{S} \models C \sqsubseteq D$ if and only if $\mathcal{O}_{T} \models C \sqsubseteq D$, and likewise for role inclusions and any other type of ontology statement supported by $\mathcal{L}_{T}$. We say that $\mathcal{O}_{T}$ is sound as an approximation if it satisfies the "if" part of this property and complete if it satisfies the "only if" part. We consider the case that

\footnotetext{
${ }^{1}$ See, for example, the BioPortal repository at https://bioportal. bioontology.org/.
}

$\mathcal{O}_{T}$ must be formulated in $\Sigma$ (non-projective approximation) and the case that additional symbols are admitted (projective approximation).

In practice, approximations are often constructed in an ad hoc way that is sound but not complete. In fact, it is common to simply drop all statements from $\mathcal{O}_{S}$ that are not expressible in $\mathcal{L}_{T}$, or at least inexpressible parts thereof. This easily leads to incompleteness, as illustrated by the following example extracted from the Galen ontology ${ }^{2}$, slightly simplified for presentation purposes. Let PathoPhen stand for "pathological phenomenon", isConOf for "is consequence of", and hasCon for "has consequence". Galen contains the following statements formulated in the DL $\mathcal{E} \mathcal{L H} \mathcal{H}$, the first three being concept inclusions and the fourth one a role inclusion:

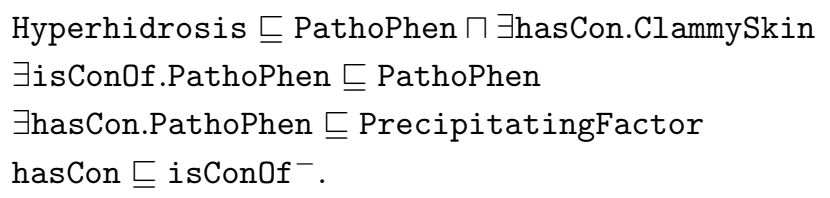

These imply as a consequence

$$
\text { Hyperhidrosis } \sqsubseteq \text { PrecipitatingFactor. }
$$

Assume that this ontology has to be approximated in the fragment $\mathcal{E} \mathcal{L H}$ of $\mathcal{E} \mathcal{L H} \mathcal{I}$ that does not admit inverse roles. A typical ad hoc approach would be to simply drop the role inclusion in the fourth line, resulting in an incomplete approximation that no longer has Consequence (1). This, however, can easily be fixed by further adding the concept inclusion

$$
\begin{array}{r}
\text { Hyperhidrosis } \sqcap \exists \text { hasCon. } \top \sqsubseteq \\
\exists \text { hasCon. } \exists \text { isConOf.Hyperhidrosis. }
\end{array}
$$

as a (partial) substitute of the dropped role inclusion.

The aim of this paper is to systematically study the structure of complete ontology approximations. There is, however, a major caveat. As we show, complete approximations must be infinite even in rather simple cases. Moreover, while finite approximations exist when the depth of the concept inclusions to be preserved is bounded by a constant, the resulting approximations are still of non-elementary size. There is no miraculous way around these facts and thus the approximations constructed in this paper cannot be directly used in

\footnotetext{
${ }^{2} \mathrm{http}: / /$ www.opengalen.org/
} 
applications. However, they provide an important theoretical foundation that enable and guide informed decisions when constructing incomplete approximations in practice. In the above example taken from Galen, for instance, concept inclusion (2) is part of the complete approximation proposed in this paper and thus an explicit candidate for inclusion also in approximations constructed in practice.

As the source DL $\mathcal{L}_{S}$, we consider the expressive Horn DL Horn- $\mathcal{S} \mathcal{R} \mathcal{I} \mathcal{F}$ and fragments thereof. As the target DL $\mathcal{L}_{T}$, we consider $\mathcal{E} \mathcal{L} \mathcal{R}_{\perp}$ and corresponding fragments thereof, where $\mathcal{E} \mathcal{L} \mathcal{R}_{\perp}$ denotes the extension of the more widely known DL $\mathcal{E} \mathcal{L H}_{\perp}$ with role inclusions of the form $r_{1} \circ \cdots \circ r_{n} \sqsubseteq r$. Subsumption is EXPTIME-complete in all considered source DLs and PTIME-complete in all cosidered target DLs [Baader et al., 2005; Krötzsch et al., 2013]. While our approximations do not aim at efficient reasoning, we thus support ontology designers who build an ontology in a tractable DL and want to import in a well-understood way from an existing ontology formulated in a computationally more expensive DL.

We provide the following results. In Section 3, we construct $\mathcal{E} \mathcal{L} \mathcal{F}$-to- $\mathcal{E} \mathcal{L}$ approximations, thus approximating away functional roles. We then proceed to $\mathcal{E} \mathcal{L} \mathcal{H} \mathcal{I}$-to- $\mathcal{E} \mathcal{L} \mathcal{H}$, approximating away inverse roles, where the $\mathcal{I}$ typeset in small font means that inverse roles are admitted only in role inclusions of the form $r \sqsubseteq s^{-}$but not in concept inclusions. This is a very common way to use inverse roles in practice, for example more than $96 \%$ of the ontologies in BioPortal that use inverse roles at all use them only in this form and this is similar for other ontology repositories. We next treat $\mathcal{E} \mathcal{L} \mathcal{H}_{\mathcal{I}} \mathcal{F}_{\perp}$ to- $\mathcal{E} \mathcal{L} \mathcal{H}_{\perp}$ under a certain syntactic assumption that restricts the interplay of role inclusions, functional roles, and inverse roles in $\mathcal{O}_{S}$. This covers also other relevant subcases such as $\mathcal{E} \mathcal{L} \mathcal{H} \mathcal{F}$-to- $\mathcal{E} \mathcal{L} \mathcal{H}$, without syntactic restrictions. All approximations constructed in Section 3 are non-projective and also provide finite approximations in the depth bounded case. The completeness proofs are non-trivial and use a version of the chase that is specifically tailored to our approximation schemes.

In Section 4, we present $\mathcal{E} \mathcal{L} \mathcal{R} \mathcal{I} \mathcal{F}_{\perp}$-to- $\mathcal{E} \mathcal{L} \mathcal{R}_{\perp}$ approximations. The presented approximations are non-projective when $\mathcal{O}_{S}$ is inverse closed, meaning that for every role name $r$ in $\mathcal{O}_{S}$, there is a role name $\hat{r}$ that is defined via role inclusions to be the inverse of $r$. This also yields projective approximations for the case where inverse closedness is not assumed and for the Horn- $\mathcal{S} \mathcal{R} \mathcal{I} \mathcal{F}$-to- $\mathcal{E} \mathcal{L} \mathcal{R}_{\perp}$ case through a wellknown normalization procedure. The completeness proof is again non-trivial, but based on a different approach, namely a novel connection between ontology approximation and the axiomatizations of quasi-equations valid in classes of semilattices with operators (SLOs) [Sofronie-Stokkermans, 2013; Sofronie-Stokkermans, 2017; Kikot et al., 2017].

We then proceed to study $\mathcal{E} \mathcal{L} \mathcal{I}_{\perp}$-to- $\mathcal{E} \mathcal{L}_{\perp}$ approximations in Section 5. In contrast to the cases considered before, where (after normalization) both $\mathcal{L}_{S}$ and $\mathcal{L}_{T}$ are based on the concept language $\mathcal{E} \mathcal{L}_{\perp}$, here the concept language of $\mathcal{L}_{S}$ is different from the one of $\mathcal{L}_{T}$. We present non-projective approximations for unrestricted ontologies $\mathcal{O}_{S}$ and for ontologies $\mathcal{O}_{S}$ which are in the well-known normal form for $\mathcal{E} \mathcal{L} \mathcal{I}_{\perp}$ ontologies that avoids syntactic nesting of concepts. The two approximation schemes are remarkably different.

In Section 6, we show that finite approximations are not guaranteed to exist and that there are cases where depth bounded approximations must be non-elementary in size.

Proof details are available in the appendix, which is available at http://www.informatik.uni-bremen.de/tdki/.

\section{Related Work}

Approximation in a DL context was first studied in [Selman and Kautz, 1996] where $\mathcal{F} \mathcal{L}$ concepts are approximated by $\mathcal{F} \mathcal{L}^{-}$concepts and in [Brandt et al., 2002] where $\mathcal{A L C}$ concepts are approximated by $\mathcal{A L E}$ concepts. In both cases, the approximation always exists, but ontologies are not considered. An incomplete approach to approximating $\mathcal{S H O I N}$ ontologies in DL-Lite $\mathcal{F}$ is presented in [Pan and Thomas, 2007] and complete (projective) approximations of $\mathcal{S R O} \mathcal{I} \mathcal{Q}$ ontologies in DL-Lite $\mathcal{A}$ are given in [Botoeva et al., 2010]. Such approximations are guaranteed to exist due to the limited expressive power of DL-Lite D. In [Lutz et al., 2012], approximation of $\mathcal{E} \mathcal{L U}$ ontologies in terms of $\mathcal{E} \mathcal{L}$ ontologies is studied, the main result being that it is EXPTIME-hard and in 2EXPTIME to decide whether a finite complete approximation exists. An incomplete ap-

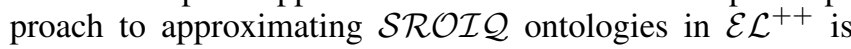
in [Ren et al., 2010]. There are also approaches towards efficient DL reasoning that involve computing approximations which are intentionally incomplete to avoid compromising efficiency [Schaerf and Cadoli, 1995; Groot et al., 2005; Carral et al., 2014]. Related to approximation is the problem whether a given $\mathcal{L}_{S}$ ontology can be equivalently rewritten into the fragment $\mathcal{L}_{T}$ of $\mathcal{L}_{S}$, either non-projectively [Lutz et al., 2011] or projectively [Konev et al., 2016]; note that this asks whether we have to approximate at all.

\section{Preliminaries}

Let $\mathrm{N}_{\mathrm{C}}$ and $\mathrm{N}_{\mathrm{R}}$ be disjoint and countably infinite sets of concept and role names. A role is a role name $r$ or an inverse

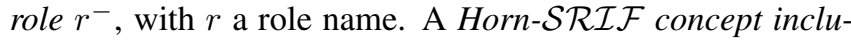
sion (CI) is of the form $L \sqsubseteq R$, where $L$ and $R$ are concepts defined by the syntax rules

$$
\begin{aligned}
R, R^{\prime}: & :=\top|\perp| A|\neg A| R \sqcap R^{\prime}|\neg L \sqcup R| \exists \rho . R \mid \forall \rho . R \\
L, L^{\prime}:: & =\top|\perp| A\left|L \sqcap L^{\prime}\right| L \sqcup L^{\prime} \mid \exists \rho . L
\end{aligned}
$$

with $A$ ranging over concept names and $\rho$ over roles. The depth of a concept $R$ or $L$ is the nesting depth of the constructors $\exists \rho$ and $\forall \rho$. For example, the concept $\exists r . B \sqcap \exists r . \exists s . A$ is of depth 2. A Horn-SRIF ontology $\mathcal{O}$ is a set of

- Horn-SRIF concept inclusions,

- functionality assertions func $(\rho)$, and

- role inclusions $(R I s) \rho_{1} \circ \cdots \circ \rho_{n} \sqsubseteq \rho$.

We adopt the standard restriction that if $n \geq 2$, then neither $\mathcal{O} \models \operatorname{func}(\rho)$ nor $\mathcal{O} \models \operatorname{func}\left(\rho^{-}\right)$. The semantics of

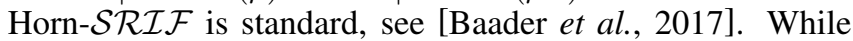
ontologies used in practice have to be finite, in this paper we shall frequently consider also infinite ontologies. W.l.o.g,, we generally assume that the $\perp$ concept occurs only in CIs of the form $C \sqsubseteq \perp$, where $C$ does not contain $\perp$. 
We briefly introduce the relevant fragments of Horn$\mathcal{S R \mathcal { F }}$, for details see [Baader et al., 2017]. An $\mathcal{E} \mathcal{L} \mathcal{I}_{\perp}$ concept is built according to the syntax rule for $L$ above, but omitting disjunction. An $\mathcal{E} \mathcal{L} \mathcal{R} \mathcal{I} \mathcal{F}_{\perp}$ ontology is a Horn$\mathcal{S R \mathcal { I F }}$ ontology in which both the left- and right-hand sides of CIs are $\mathcal{E} \mathcal{L} \mathcal{I}_{\perp}$ concepts. $\mathcal{E} \mathcal{L} \mathcal{H} \mathcal{I} \mathcal{F}_{\perp}$ is defined likewise, but admitting only role hierarchies instead of role inclusions, which take the form $r \sqsubseteq s$. Fragments of $\mathcal{E} \mathcal{L} \mathcal{R} \mathcal{I} \mathcal{F}_{\perp}$ and $\mathcal{E} \mathcal{L} \mathcal{H} \mathcal{I} \mathcal{F}_{\perp}$ can obtained by dropping expressive means that are identified by a standard naming scheme: $\mathcal{H}$ indicates role hierarchies, $\mathcal{R}$ role inclusions, $\mathcal{I}$ inverse roles, $\mathcal{F}$ functionality assertions, and $\cdot \perp$ the bottom concept. It should thus be understood, for example, what an $\mathcal{E} \mathcal{L} \mathcal{I}_{\perp}$ ontology is and what an $\mathcal{E} \mathcal{L}$ concept is. Among these DLs, $\mathcal{E} \mathcal{L} \mathcal{R}_{\perp}$ is maximal with a tractable subsumption problem. In all of the above DLs not contained in $\mathcal{E} \mathcal{L} \mathcal{R}_{\perp}$, subsumption is EXPTIME-complete [Baader et al., 2005; Baader et al., 2008].

A signature $\Sigma$ is a set of concept and role names, uniformly referred to as symbols. When speaking of $\mathcal{E} \mathcal{L}(\Sigma)$ concept, we mean $\mathcal{E} \mathcal{L}$ concepts that only use symbols from $\Sigma$, and likewise for other DLs. We use $\operatorname{sig}(\mathcal{O})$ to denote the set of symbols used in ontology $\mathcal{O}$.

We now define the central notions of this paper.

Definition 1 Let $\mathcal{O}_{S}$ be a Horn-SRIF ontology with $\operatorname{sig}\left(\mathcal{O}_{S}\right)=\Sigma$ and let $\mathcal{L}_{T}$ be any of the fragments of Horn$\mathcal{S R I \mathcal { F }}$ introduced above. A (potentially infinite) $\mathcal{L}_{T}$ ontology $\mathcal{O}_{T}$ is an $\mathcal{L}_{T}$ approximation of $\mathcal{O}_{S}$ if

$$
\mathcal{O}_{S} \models \alpha \text { iff } \mathcal{O}_{T} \models \alpha
$$

for all concept inclusions, role inclusions, and functionality assertions $\alpha$ that fall within $\mathcal{L}_{T}$ and use only symbols from $\Sigma$. We say that $\mathcal{O}_{T}$ is non-projective if $\operatorname{sig}\left(\mathcal{O}_{T}\right) \subseteq \Sigma$ and projective otherwise.

For $\ell \in \mathbb{N} \cup\{\omega\}$, (non-projective and projective) $\ell$ bounded $\mathcal{L}_{T}$ approximations are defined in the same way except that only concept inclusions $\alpha=C \sqsubseteq D$ are considered where $C$ and $D$ are of depth bounded by $\ell$.

Note that $\omega$-bounded approximations are identical to unbounded approximations, we use the term only for uniformity. Trivially, infinite (non-projective and projective) approximations always exist: take as $\mathcal{O}_{T}$ the set of all inclusions and assertions from $\mathcal{L}_{T}$ that are entailed by $\mathcal{O}_{S}$. One can show that there are $\mathcal{E} \mathcal{L} \mathcal{I}$ ontologies $\mathcal{O}_{S}$ that have a finite projective $\mathcal{E} \mathcal{L}$ approximation, but no finite non-projective $\mathcal{E} \mathcal{L}$ approximation; details are in the appendix.

Example 1 Consider the $\mathcal{E} \mathcal{L} \mathcal{F}$ ontology

$$
\begin{aligned}
\mathcal{O}_{S}=\{ & \exists \text { hasSupervisor. } \top \sqsubseteq \text { Employee, } \\
& \text { func(reportsTo })\} .
\end{aligned}
$$

There is no finite $\mathcal{E} \mathcal{L}$ approximation since for all $n, m \geq 1$, $\mathcal{O}_{S}$ entails the $\mathcal{E} \mathcal{L}$ concept inclusion

$$
\begin{array}{r}
\exists \text { reportsTo. } \text { hasSupervisor }^{n} \cdot \top \sqcap \exists_{\text {reportsTo }}{ }^{m} \cdot \top \\
\sqsubseteq \exists \text { reportsTo.( }\left(\exists \text { hasSupervisor }{ }^{n} \cdot \top\right. \\
\left.\sqcap \exists \text { reportsTo }{ }^{m-1} \cdot \top\right) .
\end{array}
$$

In practice, it clearly does not make sense to include all these CIs in the approximation. Similarly to the example in the introduction, though, it may pay off to include some of them. Choosing the right ones requires a careful inspection of the ontology and application at hand.

With $\mathcal{L}_{S}$-to- $\mathcal{L}_{T}$ approximation, $\mathcal{L}_{S}$ an ontology language and $\mathcal{L}_{T}$ a fragment thereof, we mean the task of approximating an $\mathcal{L}_{S}$ ontology in $\mathcal{L}_{T}$. We call $\mathcal{L}_{S}$ the source $D L$ and $\mathcal{L}_{T}$ the target $D L$.

An alternative definition of approximations is obained by dropping the restriction that $\alpha$ can use only symbols from $\Sigma$. We do not use that definition because then even in the 1-bounded case, finite approximations might not exist.

Example 2 Take the $\mathcal{E} \mathcal{L} \mathcal{I}$ ontology $\mathcal{O}_{S}=\left\{\exists r^{-} . A \sqsubseteq B\right\}$. Then $\mathcal{O} \models A \sqcap \exists r . X \sqsubseteq \exists r .(B \sqcap X)$ for each of the infinitely many concept names $X \in \mathrm{N}_{\mathrm{C}}$. Thus, every (projective or non-projective) 1-bounded $\mathcal{E} \mathcal{L}$ approximation of $\mathcal{O}_{S}$ must be infinite under the alternative definition of approximation.

We now make some basic observations regarding approximations. The proof is straightforward.

Lemma 1 Let $\mathcal{O}_{S}$ be a Horn-SRIF ontology with

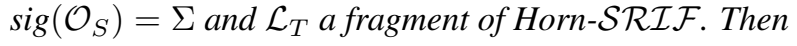

1. $\mathcal{O}_{T}$ is an $\mathcal{L}_{T}$ approximation of $\mathcal{O}_{S}$ iff $\mathcal{O}_{S}=\mathcal{O}_{T}$ and for every $\mathcal{L}_{T}$ ontology $\mathcal{O}$ with $\mathcal{O}_{S} \models \mathcal{O}$ and $\operatorname{sig}(\mathcal{O}) \subseteq \Sigma$, $\mathcal{O}_{T}=\mathcal{O}$;

2. $\bigcup_{i \geq 0} \mathcal{O}_{\ell}$ is an $\mathcal{L}_{T}$ approximation of $\mathcal{O}_{S}$ if for all $\ell \geq 0$, $\mathcal{O}_{\ell}$ is an $\ell$-bounded $\mathcal{L}_{T}$ approximation of $\mathcal{O}_{S}$; the same is true for projective $\mathcal{L}_{T}$ approximations provided that $\operatorname{sig}\left(\mathcal{O}_{\ell}\right) \cap \operatorname{sig}\left(\mathcal{O}_{\ell^{\prime}}\right) \subseteq \Sigma$ when $\ell \neq \ell^{\prime}$.

Point 1 may be viewed as an alternative definition of (nonprojective) approximations. Point 2 is important because it sometimes allows us to concentrate on bounded approximations in proofs. The following is well-known, see for example [Bienvenu et al., 2016].

Lemma 2 Given a Horn-SRIF ontology $\mathcal{O}_{S}$ with $\operatorname{sig}(\mathcal{O})=\Sigma$, one can construct in polynomial time an $\mathcal{E} \mathcal{L} \mathcal{R} \mathcal{I} \mathcal{F}_{\perp}$ ontology $\mathcal{O}_{S}^{\prime}$ with $\operatorname{sig}\left(\mathcal{O}_{S}^{\prime}\right) \supseteq \Sigma$ that entails the

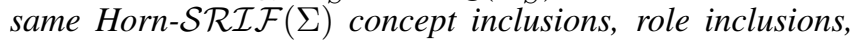
and functionality assertions.

The construction of the ontology $\mathcal{O}_{S}^{\prime}$ from Lemma 2 requires the introduction of fresh concept names. Still, every $\ell-$ bounded $\mathcal{L}_{T}$ approximation of $\mathcal{O}_{S}^{\prime}$ is a projective $\ell$-bounded $\mathcal{L}_{T}$ approximation of $\mathcal{O}_{S}$.

\section{Depth Bounded Approximation}

The goal of this section is to study non-projective approximations in various DLs, both in the unbounded case and in the depth bounded case. We start with approximating away functionality assertions, then inverse roles, and finally their combination (assuming a certain syntactic restriction), also admitting role hierarchies and the bottom concept. This stepby-step approach aims to facilitate presentation and in fact the final theorem in this section subsumes the earlier ones and is the only one that we prove explicitly.

We start with $\mathcal{E} \mathcal{L} \mathcal{F}$-to- $\mathcal{E} \mathcal{L}$ approximation. Let $C$ be an $\mathcal{E} \mathcal{L}$ concept and $k \geq 0$. A leaf occurrence of a concept name $A$ in $C$ means an occurrence of $A$ inside a conjunction that 
does not contain conjuncts of the form $\exists$ r.D. For example, all occurrences of $A$ in $A \sqcap B$ and in $B \sqcap \exists r .(A \sqcap B)$ are leaf occurrences, but the occurrence of $A$ in $A \sqcap B \sqcap \exists r . \top$ is not. By decorating $C$ with subconcepts from $\mathcal{O}_{S}$ at leaves, we mean to replace any number of leaf occurrences of a concept name $A$ by a concept $A \sqcap D_{1} \sqcap \cdots \sqcap D_{k}$ where $D_{1}, \ldots, D_{k}$ are concepts that occur in $\mathcal{O}_{S}$, possibly as subconcepts.

Theorem 1 Let $\mathcal{O}_{S}$ be an $\mathcal{E} \mathcal{L} \mathcal{F}$ ontology, $\Sigma=\operatorname{sig}\left(\mathcal{O}_{S}\right)$, and $\ell \in \mathbb{N} \cup\{\omega\}$ a depth bound. Define $\mathcal{O}_{T}$ to be the $\mathcal{E} \mathcal{L}$ ontology that contains:

1. all CIs from $\mathcal{O}_{S}$;

2. all CIs $\exists r . C_{1} \sqcap \exists r . C_{2} \sqsubseteq \exists r .\left(C_{1} \sqcap C_{2}\right)$ such that func $(r) \in \mathcal{O}_{S}$ and $C_{1}, C_{2}$ are $\mathcal{E} \mathcal{L}(\Sigma)$ concepts of depth bounded by $\max \{0, \ell-1\}$ decorated with subconcepts of $\mathcal{O}_{S}$ at leaves.

Then $\mathcal{O}_{T}$ is an $\ell$-bounded approximation of $\mathcal{O}_{S}$.

Note that the construction of $\mathcal{O}_{T}$ is entirely syntactic, that is, it involves no reasoning. Due to Point $2, \mathcal{O}_{T}$ is infinite when $\ell=\omega$. On the other hand, $\mathcal{O}_{T}$ is finite when $\ell<\omega$ and thus Theorem 1 proves the existence of finite depth bounded non-projective approximations in the $\mathcal{E} \mathcal{L} \mathcal{F}$-to- $\mathcal{E} \mathcal{L}$ case. It is interesting to remark that Theorem 1 also reproves the upper bound for subsumption in $\mathcal{E} \mathcal{L} \mathcal{F}$ : first extend the ontology so that it suffices to decide subsumption between concept names, then compute the 0-bounded approximation which is of single exponential size, and then decide subsumption in $\mathcal{E} \mathcal{L}$ in PTIME [Baader et al., 2005]. Note that for $\ell=0$, the concepts $C_{1}, C_{2}$ in Point 2 of Theorem 1 are simply conjunctions of subconcepts from $\mathcal{O}_{S}$.

We next consider inverse roles. Here, the most basic case is that inverse roles can occur in role inclusions, but not in concepts. As noted in the introduction, this case actually occurs rather frequently in practice. To indicate the restricted use of inverse roles, we typeset the $\mathcal{I}$ in a smaller font, as in $\mathcal{E} \mathcal{L} \mathcal{H}_{\mathcal{I}} \mathcal{F}$ and $\mathcal{E} \mathcal{L R}_{\mathcal{I}} \mathcal{F}$. The most basic case is now that of $\mathcal{E} \mathcal{L} \mathcal{H} \mathcal{I}$-to- $\mathcal{E} \mathcal{L} \mathcal{H}$ approximation. We assume w.l.o.g. that role inclusions only take the two forms $r \sqsubseteq s$ and $r \sqsubseteq s^{-}$.

Theorem 2 Let $\mathcal{O}_{S}$ be an $\mathcal{E} \mathcal{L} \mathcal{H}$ ontology, $\Sigma=\operatorname{sig}\left(\mathcal{O}_{S}\right)$, and $\ell \in \mathbb{N} \cup\{\omega\}$ a depth bound. Define $\mathcal{O}_{T}$ to be the $\mathcal{E} \mathcal{L} \mathcal{H}$ ontology that contains, for $\ell^{\prime}=\max \{0, \ell-1\}$ :

1. all CIs from $\mathcal{O}_{S}$;

2. all RIs $r \sqsubseteq s$ such that $\mathcal{O}_{S} \models r \sqsubseteq s$ and role names $r, s$ occur in $\overline{\mathcal{O}}_{S}$;

3. all CIs $C_{1} \sqcap \exists r . C_{2} \sqsubseteq \exists r .\left(C_{2} \sqcap \exists s . C_{1}\right)$ such that $\mathcal{O}_{S} \models$ $r \sqsubseteq s^{-}, \exists s . C_{1}$ is a subconcept of $\mathcal{O}_{S}$ or an $\mathcal{E} \mathcal{L}(\Sigma)$ concept of depth bounded by $\ell$, and $C_{2}$ is an $\mathcal{E} \mathcal{L}(\Sigma)$ concept of depth bounded by $\ell^{\prime}$ decorated with subconcepts of $\mathcal{O}_{S}$ at leaves.

Then $\mathcal{O}_{T}$ is an $\ell$-bounded approximation of $\mathcal{O}_{S}$.

Note that Point 2 is not entirely syntactic, but involves reasoning. It is easy to see and well-known, however, that in $\mathcal{E} \mathcal{L} \mathcal{I}$ deciding whether $\mathcal{O}_{S}=r \sqsubseteq s$ is in PTIME.

We now consider the $\overline{\mathcal{E}} \mathcal{L} \mathcal{H} \mathcal{I}_{\perp}$-to- $\mathcal{E} \mathcal{L} \mathcal{H}_{\perp}$ case which combines functional and inverse roles. It turns out that there are subtle interactions between functional, inverse roles, and role hierarchies which we tame by making the following assumption:
(D) $\mathcal{O}_{S}=r \sqsubseteq s^{-}$implies that neither func $(s) \in \mathcal{O}_{S}$ nor $\operatorname{func}\left(s^{-}\right) \in \mathcal{O}_{S}$.

The next theorem is the main result of this section.

Theorem 3 Let $\mathcal{O}_{S}$ be an $\mathcal{E} \mathcal{L} \mathcal{H} \mathcal{F}_{\perp}$ ontology that satisfies $(\mathbb{\nabla}), \Sigma=\operatorname{sig}\left(\mathcal{O}_{S}\right)$, and $\ell \in \mathbb{N} \cup\{\omega\}$ a depth bound. Define $\mathcal{O}_{T}$ to be the $\mathcal{E} \mathcal{L} \mathcal{H}_{\perp}$ ontology that contains, for $\ell^{\prime}=$ $\max \{0, \ell-1\}$ :

1. all CIs from $\mathcal{O}_{S}$;

2. all $r \sqsubseteq s$ such that $\mathcal{O}_{S} \models r \sqsubseteq s, r, s$ role names that occur in $\mathcal{O}_{S}$;

3. all CIs $C_{1} \sqcap \exists r . C_{2} \sqsubseteq \exists r .\left(C_{2} \sqcap \exists s . C_{1}\right)$ such that $\mathcal{O}_{S} \models$ $r \sqsubseteq s^{-}, \exists s . C_{1}$ is a subconcept of $\mathcal{O}_{S}$ or an $\mathcal{E} \mathcal{L}(\Sigma)$ concept of depth bounded by $\ell$, and $C_{2}$ is an $\mathcal{E} \mathcal{L}(\Sigma)$ concept of depth bounded by $\ell^{\prime}$ decorated with subconcepts of $\mathcal{O}_{S}$ at leaves;

4. all CIs $\exists r_{1} . C_{1} \sqcap \exists r_{2} . C_{2} \sqsubseteq \exists r_{1} .\left(C_{1} \sqcap C_{2}\right)$ such that there is a role name $s$ with $\mathcal{O}_{S} \models r_{1} \sqsubseteq s, \mathcal{O}_{S} \models r_{2} \sqsubseteq s$, and func $(s) \in \mathcal{O}_{S}$, and $C_{1}, C_{2}$ are $\mathcal{E} \mathcal{L}(\Sigma)$ concepts of depth bounded by $\ell^{\prime}$ decorated with subconcepts of $\mathcal{O}_{S}$ at leaves.

Then $\mathcal{O}_{T}$ is an $\ell$-bounded approximation of $\mathcal{O}_{S}$.

It is not hard to see that Theorem 3 implies Theorems 1 and 2. It also settles additional approximation cases such as $\mathcal{E} \mathcal{L} \mathcal{H} \mathcal{F}_{\perp}$-to- $\mathcal{E} \mathcal{L} \mathcal{H}_{\perp}$, without syntactic assumptions. Points 2 to 4 require deciding whether $\mathcal{O}_{S}=r \sqsubseteq s^{(-)}$, which is EXPTIME-complete in $\mathcal{E} \mathcal{L} \mathcal{H} \mathcal{I} \mathcal{F}_{\perp}$ as can be proved by mutual reduction with subsumption.

It is straightforward to verify that the ontology $\mathcal{O}_{T}$ constructed in Theorem 3 is sound as an approximation, that is, $\mathcal{O}_{S} \models \mathcal{O}_{T}$. Completeness is non-trivial. It is established by first introducing a version of the chase that is closely tailored towards the construction of $\mathcal{O}_{T}$ given in Theorem 3, then showing that the chase is sound and complete regarding the derivation of $\mathcal{E} \mathcal{L}(\Sigma)$ CIs of depth bounded by $\ell$, and finally proving that the CIs in $\mathcal{O}_{T}$ can simulate derivations of the chase. With chase, we mean a rule based approach to constructing (infinite) 'canonical models' [Kontchakov and Zakharyaschev, 2014].

An interesting observation about the proof of Theorem 3 is that it actually yields a more general result than stated in that theorem. Instead of $\ell$-bounded approximations, one could define $\Gamma$-bounded approximations for any set of $\mathcal{E} \mathcal{L}_{\perp}$-concepts $\Gamma$ closed under subconcepts, that is, only concepts from $\Gamma$ are considered in concept inclusions $\alpha$ in Definition 1. We then obtain a version of Theorem 3 in which "concept of depth bounded by $\ell$ or $\ell^{\prime}$ " is replaced with "concept from $\Gamma$ " (decorated with subconcepts of $\mathcal{O}_{S}$ at leaves as needed). While one could choose for $\Gamma$ the set of all concepts of depth bounded by some $\ell$, other choices of $\Gamma$ might be natural, too. For example, if one wants to decide subsumption between compound $\mathcal{E} \mathcal{L}$ concepts $C$ and $D$ relative to an $\mathcal{E} \mathcal{L} \mathcal{H} \mathcal{I}$ ontology $\mathcal{O}_{S}$ without resorting to concept names, then one can approximate $\mathcal{O}_{S}$ in $\mathcal{E} \mathcal{L}$ relative to the set $\Gamma$ of subconcepts of $C$ and $D$ and then check whether $\mathcal{O}_{T}$ entails $C \sqsubseteq D$. While this is clearly not efficient in practice, it raises the interesting question of how to identify sets $\Gamma$ that are tailored towards the actual application of an ontology. 
We now briefly discuss the case in which the restriction $(\nabla)$ is dropped. One can prove that we then need to extend Points 1 to 4 of Theorem 3 with the following:

5. all CIs $\exists r . \exists s . C \sqsubseteq C$ such that $\mathcal{O}_{S} \models r \sqsubseteq s^{-}$, func $(s) \in \mathcal{O}_{S}$, and $C$ is a subconcept of $\mathcal{O}_{S}$ or an $\mathcal{E} \mathcal{L}(\Sigma)$ concept of depth bounded by $\ell ;$

6. all CIs $\exists s . \exists r . C \sqsubseteq C$ such that $\mathcal{O}_{S} \models r \sqsubseteq s^{-}$, func $\left(s^{-}\right) \in \mathcal{O}_{S}$, and $C$ is a subconcept of $\mathcal{O}_{S}$ or an $\mathcal{E} \mathcal{L}(\Sigma)$ concept of depth bounded by $\ell$.

However, this is still not sufficient to obtain a complete approximation. Consider the $\mathcal{E} \mathcal{L} \mathcal{H} \mathcal{I}$ ontology

$$
\begin{aligned}
\mathcal{O}_{S}=\{A & \sqsubseteq \exists r_{1} \cdot \exists r_{2} \cdot(B \sqcap \exists s . \top), \\
s & \left.\sqsubseteq r_{1}, s \sqsubseteq r_{2}^{-}, \operatorname{func}\left(r_{1}^{-}\right), \operatorname{func}\left(r_{2}^{-}\right)\right\} .
\end{aligned}
$$

It can be verified that $\mathcal{O}_{S} \models A \sqsubseteq B$. However, it can also be proved that even when $\mathcal{O}_{T}$ is the set of all statements from Points 1 to 6 with $\ell=0, \mathcal{O}_{T} \forall \models A \sqsubseteq B$. It remains open whether a transparent (non-projective) approximation is possible when $(\nabla)$ is dropped.

\section{Unbounded Approximation}

We provide a significant extensions of Theorem 3 for the case of unbounded approximations, using an entirely different strategy for the completeness proof. In particular, we do not assume the $(\nabla)$ restriction adopted in Theorem 3, admit inverse roles also in concepts, and add general role inlusions both to the source and target DL, that is, we consider $\mathcal{E} \mathcal{L} \mathcal{R} \mathcal{I} \mathcal{F}_{\perp}$-to- $\mathcal{E} \mathcal{L} \mathcal{R}_{\perp}$ approximation. There is a small price that we have to pay for this generality: the approximations constructed here are projective as for every role name from the original ontology, they contain a (potentially fresh) role name that represents its inverse. It is remarkable that this rather mild form of projectiveness overcomes several problems from the purely non-projective case.

An $\mathcal{E} \mathcal{L} \mathcal{R} \mathcal{I} \mathcal{F}_{\perp}$ ontology $\mathcal{O}$ is inverse closed, that is, for every role name $r$ in $\mathcal{O}$, there is a role name $\hat{r}$ such that $\mathcal{O}$ contains $r \sqsubseteq \hat{r}^{-}$and $\hat{r} \sqsubseteq r^{-}$. We provide non-projective approximations under the assumption that the source ontology is inverse closed. This also yields projective approximations for source ontologies that are not inverse closed because we can first extend $\mathcal{O}_{S}$ with the required role names $\hat{r}$ and then approximate. Note that in practice, there are relevant examples of ontologies that are inverse closed such as Galen. If our source is inverse closed, we can further assume that there are no other occurrences of inverse roles in $\mathcal{O}_{S}$, neither in concept inclusions nor in other role inclusions. In other words, our source ontology is formulated in $\mathcal{E} \mathcal{L R}_{\mathcal{I}} \mathcal{F}_{\perp}$.

Theorem 4 Let $\mathcal{O}_{S}$ be an inverse closed $\mathcal{E} \mathcal{L} \mathcal{R}_{\mathcal{I}} \mathcal{F}_{\perp}$ ontology and $\Sigma=\operatorname{sig}\left(\mathcal{O}_{S}\right)$. Define $\mathcal{O}_{T}$ to be the $\mathcal{E} \mathcal{L} \mathcal{R}_{\perp}$ ontology that contains for all $\mathcal{E} \mathcal{L}(\Sigma)$ concepts $C, D$ :

1. all CIs in $\mathcal{O}_{S}$;

2. all RIs $r \sqsubseteq s$ with $\mathcal{O}_{S} \models r \sqsubseteq s, r, s \in \Sigma$ role names;

3. all RIs $r_{1} \circ \cdots \circ r_{n} \sqsubseteq r$ and $\hat{r}_{n} \circ \cdots \circ \hat{r}_{1} \sqsubseteq \hat{r}$ such that $r_{1} \circ \cdots \circ r_{n} \sqsubseteq r \in \mathcal{O}_{S}$ with $n \geq 2$;

4. all CIs $C \sqcap \exists r . D \sqsubseteq \exists r .(D \sqcap \exists \hat{r} . C)$;
5. all CIs $\exists r . C \sqcap \exists r . D \sqsubseteq \exists r .(C \sqcap D)$ such that func $(r) \in \mathcal{O}_{S}$;

6. all CIs $\exists r . \exists \hat{r} . C \sqsubseteq C$ such that func $(\hat{r}) \in \mathcal{O}_{S}$.

Then $\mathcal{O}_{T}$ is an $\mathcal{E} \mathcal{L} \mathcal{R}_{\perp}$ approximation of $\mathcal{O}_{S}$.

Note that Points 1 to 3 essentially take over the part of $\mathcal{O}_{S}$ that is expressible in $\mathcal{E} \mathcal{L} \mathcal{R}_{\perp}$, Point 4 aims at capturing the consequences of inverse roles, Point 5 at functional roles, and Point 6 at the interaction between functional roles and inverse roles. Points 4 to 6 all introduce infinitely many CIs. Via Lemma 2, Theorem 4 also yields projective approximations for the Horn- $\mathcal{S} \mathcal{R} \mathcal{I} \mathcal{F}$-to- $\mathcal{E} \mathcal{L} \mathcal{R}_{\perp}$ case.

The following example shows that the CIs in Point 5 of Theorem 3, which unlike Point 5 of Theorem 4 mix functional roles and role inclusions, are implied by the ontology $\mathcal{O}_{T}$ constructed in Theorem 4. The example also illustrates the strength of the 'inverse closed' property.

Example 3 Let $r_{1} \sqsubseteq s, r_{2} \sqsubseteq s$, func $(s) \in \mathcal{O}_{S}$, and let $C_{1}, C_{2}$ be $\mathcal{E} \mathcal{L}$-concepts. We aim to show that

$$
\mathcal{O}_{T} \equiv \exists r_{1} \cdot C_{1} \sqcap \exists r_{2} \cdot C_{2} \sqsubseteq \exists r_{1} \cdot\left(C_{1} \sqcap C_{2}\right) .
$$

Due to Points 2 and 5 in Theorem 4, it suffices to show that

$$
\mathcal{O}_{T} \models \exists s . C_{i} \sqcap \exists r_{i} . \top \sqsubseteq \exists r_{i} . C_{i} \text { for } i \in\{1,2\} .
$$

This CI, in turn, can be proved from $\mathcal{O}_{T}$ as follows:

$$
\begin{aligned}
\mathcal{O}_{T}= & \exists s . C_{i} \sqcap \exists r_{i} \cdot \top & & \\
& \sqsubseteq \exists r_{i} \cdot\left(\top \sqcap \exists \hat{r}_{i} \cdot \exists s . C_{i}\right) & & (\text { Point } 4) \\
& \sqsubseteq \exists r_{i} \cdot\left(\top \sqcap \exists \hat{s} \cdot \exists s . C_{i}\right) & & \left(\hat{r}_{i} \sqsubseteq \hat{s}_{i} \in \mathcal{O}_{T} \text { by Point } 2\right) \\
& \sqsubseteq \exists r_{i} \cdot\left(\top \sqcap C_{i}\right) & & (\text { Point } 6) .
\end{aligned}
$$

It is straightforward to show that the ontology $\mathcal{O}_{T}$ from Theorem 4 is sound as an approximation. To prove completeness, we establish a novel connection between $\mathcal{E} \mathcal{L}_{\perp}$ approximations and axiomatizations of the quasi-equations that are valid in classes of semilattices with operators (SLOs) [Jackson, 2004; Sofronie-Stokkermans, 2017; Kikot et al., 2017]. Roughly speaking, an approximation is obtained from such an axiomatization by instantiating its equations, which correspond (in the sense of modal correspondence theory) to the role inclusions in the original ontology, with $\mathcal{E} \mathcal{L}$ concepts.

\section{Inverse Roles in Concept Inclusions}

As discussed before Theorem 4, the approximations provided by that theorem also cover the case where inverse roles are admitted in concept inclusions. This is achieved, however, by first making the ontology inverse closed and then dropping inverse roles from CIs. Here, we investigate alternative approaches in the basic case of $\mathcal{E} \mathcal{L} \mathcal{I}_{\perp}$-to- $\mathcal{E} \mathcal{L}_{\perp}$, both nonprojectively and projectively, in the latter case using a wellknown normal form for $\mathcal{E} \mathcal{L} \mathcal{I}_{\perp}$ ontologies that avoids syntactic nesting [Baader et al., 2017].

A key to constructing non-projective approximations is the observation that concepts of the form $\exists r^{-} . C$ can be used as a marker that is invisible to $\mathcal{E} \mathcal{L}_{\perp}$.

Example 4 Let $\mathcal{O}_{S}=\left\{A \sqsubseteq \exists s^{-} . \top, \exists r^{-} . \exists s^{-} \cdot \top \sqsubseteq\right.$ $\left.\exists s^{-} . \top, \exists s^{-} . \top \sqsubseteq B\right\}$. Then $\mathcal{O}_{S} \bar{\models}=C \sqsubseteq C^{\prime}$ for all $\mathcal{E} \mathcal{L}$ concepts $C, C^{\prime}$ where $C^{\prime}$ is obtained from $\bar{C}$ by decorating with 
$B$ any node that is reachable in $C$ from a node decorated with $A$ along an r-path (we view an $\mathcal{E} \mathcal{L}$ concept as a tree in the standard way, see for example [Konev et al., 2018]).

We now give a non-projective approximation that captures the effect demonstrated in Example 4. For an $\mathcal{E} \mathcal{L} \mathcal{I}_{\perp}$ ontology $\mathcal{O}_{S}$, let $\operatorname{cl}_{\mathcal{E} \mathcal{L}}\left(\mathcal{O}_{S}\right)$ denote the set of all $\mathcal{E} \mathcal{L}$ concepts that can be obtained by starting with a subconcept of a concept from $\mathcal{O}_{S}$ and then replacing every subconcept of the form $\exists r^{-} . D$ with $\top$. Let $C$ be an $\mathcal{E} \mathcal{L}$ concept. An $\mathcal{E} \mathcal{L}$ concept $C^{\prime}$ is a $\operatorname{cl}_{\mathcal{E} \mathcal{L}}\left(\mathcal{O}_{S}\right)$ decoration of $C$ if it can be obtained from $C$ by conjunctively adding concepts from $\mathrm{cl}_{\mathcal{E} \mathcal{L}}\left(\mathcal{O}_{S}\right)$ to a single occurrence of a subconcept in $C$.

Theorem 5 Let $\mathcal{O}_{S}$ be an $\mathcal{E} \mathcal{L} \mathcal{I}_{\perp}$ ontology and $\Sigma=$ $\operatorname{sig}\left(\mathcal{O}_{S}\right)$. Define $\mathcal{O}_{T}$ to be the $\mathcal{E} \mathcal{L}_{\perp}$ ontology $\mathcal{O}_{T}$ that contains for all $\mathcal{E} \mathcal{L}(\Sigma)$ concepts $C$ :

1. all CIs $C \sqsubseteq C^{\prime}$ such that $\mathcal{O}_{S} \models C \sqsubseteq C^{\prime}, C^{\prime} a$ $\mathrm{cl}_{\mathcal{E} \mathcal{L}}\left(\mathcal{O}_{S}\right)$ decoration of $C$;

2. all CIs $C \sqsubseteq \perp$ such that $\mathcal{O}_{S} \models C \sqsubseteq \perp$.

Then $\mathcal{O}_{T}$ is an $\mathcal{E} \mathcal{L}_{\perp}$ approximation of $\mathcal{O}_{S}$.

We prove completeness by a chase based approach. The CIs in Theorem 5 are rather different from those that we have used in Sections 3 and 4 to deal with inverse roles. Being much less constrained, they provides less guidance for constructing approximations in practice. We next observe that we can get back to the more constrained CI scheme for inverse roles by assuming the source ontology $\mathcal{O}_{S}$ to be in normal form, that is, all CIs in $\mathcal{O}_{S}$ have one of the forms $\top \sqsubseteq A_{1}$, $A_{1} \sqsubseteq \perp, A_{1} \sqsubseteq \exists \rho . A_{2}, \exists \rho . A_{1} \sqsubseteq B$, and $A_{1} \sqcap \cdots \sqcap A_{n} \sqsubseteq B$ where $A_{1}, \ldots, A_{n}, B$ range over concept names and $\rho$ ranges over roles. Every $\mathcal{E} \mathcal{L} \mathcal{I}_{\perp}$ ontology $\mathcal{O}_{S}$ can be converted into an $\mathcal{E} \mathcal{L} \mathcal{I}_{\perp}$ ontology $\mathcal{O}_{S}^{\prime}$ in normal form in linear time such that $\mathcal{O}_{S}^{\prime}$ is a conservative extension of $\mathcal{O}_{S}$ [Baader et al., 2017]. Clearly, any approximation of $\mathcal{O}_{S}^{\prime}$ is then a projective approximation of $\mathcal{O}_{S}$.

Theorem 6 Let $\mathcal{O}_{S}$ be an $\mathcal{E} \mathcal{L} \mathcal{I}_{\perp}$ ontology in normal form, $\Sigma=\operatorname{sig}\left(\mathcal{O}_{S}\right)$, and $\ell \in \mathbb{N} \cup\{\omega\}$ a depth bound. Define $\mathcal{O}_{T}$ to be the $\mathcal{E} \mathcal{L}_{\perp}$ ontology $\mathcal{O}_{T}$ that contains:

1. all CIs from $\mathcal{O}_{S}$ that are of the form $\top \sqsubseteq A, A \sqsubseteq \perp$, $\exists r . A \sqsubseteq B$, or $A \sqsubseteq \exists r . B$,;

2. all CIs $A_{1} \sqcap \cdots \sqcap A_{n} \sqsubseteq B$ such that $\mathcal{O}_{S} \models A_{1} \sqcap \cdots \sqcap$ $A_{n} \sqsubseteq B, A_{1}, \ldots, A_{n}, \bar{B} \in \mathrm{N}_{\mathrm{C}}$ occur in $\mathcal{O}_{S}$;

3. all CIs $A \sqcap \exists r . C \sqsubseteq \exists r .(C \sqcap B)$ such that $\exists r^{-} . A \sqsubseteq$ $B \in \mathcal{O}_{S}$ and $C$ is an $\mathcal{E} \mathcal{L}(\Sigma)$ concept of depth bounded by $\ell-1$.

Then $\mathcal{O}_{T}$ is an $\ell$-bounded $\mathcal{E} \mathcal{L}_{\perp}$ appoximation of $\mathcal{O}_{S}$.

It is straightforward to verify that $\mathcal{O}_{T}$ is sound. To prove completeness, we again use a chase based strategy.

\section{Size of Approximations}

We prove that finite approximations are not guaranteed to exist and that depth bounded approximations can be nonelementary in size. These results hold both for projective and non-projective approximations and for all combinations of source and target DL considered in this paper. The ontologies used to prove these results are simple and show that also for most ontologies that occur in practical applications, neither finite (complete) approximations nor depth bounded (complete) approximations of elementary size can be expected. We focus on the cases $\mathcal{E} \mathcal{L} \mathcal{I} \mathcal{H}$-to- $\mathcal{E} \mathcal{L} \mathcal{H}, \mathcal{E} \mathcal{L} \mathcal{H} \mathcal{F}$-to- $\mathcal{E} \mathcal{L} \mathcal{H}$, and $\mathcal{E} \mathcal{L} \mathcal{H} \mathcal{I}$-to- $\mathcal{E} \mathcal{L} \mathcal{H}$, starting with unbounded approximations.

Theorem 7 None of the ontologies

$$
\left\{\exists r^{-} . A \sqsubseteq B\right\}, \quad\{\operatorname{func}(r), A \sqsubseteq A\}, \quad\left\{r \sqsubseteq s^{-}, A \sqsubseteq A\right\}
$$

has finite projective $\mathcal{E} \mathcal{L} \mathcal{H}$ approximations.

To get an idea of the proof, consider $\mathcal{O}_{S}=\left\{\exists r^{-} . A \sqsubseteq B\right\}$ and let $\mathcal{O}_{T}$ be a projective $\mathcal{E} \mathcal{L} \mathcal{H}$ approximation of $\mathcal{O}_{S}$. For all $n \geq 0$, let $C_{n}=\exists r^{n}$. $\top$, where $\exists r^{n}$ denotes $n$-fold nesting of an existential restriction, and observe that

$$
\mathcal{O}_{S} \models A \sqcap \exists r . C_{n} \sqsubseteq \exists r .\left(B \sqcap C_{n}\right) .
$$

To establish the desired result, we prove that for every $n \geq 0$, there is a subconcept $M_{n}$ of $\mathcal{O}_{T}$ such that $\mathcal{O}_{T} \models M_{n} \sqsubseteq C_{n}$ and $\mathcal{O}_{T} \not \models M_{n} \sqsubseteq C_{m}$ for any $m>n$.

We next show that bounded depth approximations can be non-elementary in size. The function tower $: \mathbb{N} \times \mathbb{N} \rightarrow \mathbb{N}$ is defined as tower $(0, n):=n$ and tower $(k+1, n):=$ $2^{\text {tower }(k, n)}$. The size of a (finite) ontology is the number of symbols needed to write it, with concept and role names counting as one. We use $\Gamma_{n}$ to denote a fixed finite tautological set of $\mathcal{E} \mathcal{L}$ concept inclusions that contains the symbols $\Sigma_{n}=\left\{r_{1}, r_{2}, A_{1}, \hat{A}_{1}, \ldots, A_{n}, \hat{A}_{n}\right\}$.

Theorem 8 Let $n \geq 0$ and let $\mathcal{O}_{n}$ be the union of $\Gamma_{n}$ with any of the following sets:

$$
\left\{\exists r^{-} . A \sqsubseteq B\right\}, \quad\{\operatorname{func}(r), A \sqsubseteq A\}, \quad\left\{r \sqsubseteq s^{-}, A \sqsubseteq A\right\}
$$

For every $\ell \geq 1$, any $\ell$-bounded projective $\mathcal{E} \mathcal{L} \mathcal{H}$ approximation $\mathcal{O}_{T}$ of $\mathcal{O}_{n}$ must be of size at least tower $(\ell, n)$.

\section{Conclusion}

It remains an open problem to develop informative nonprojective approximations for (unrestricted) $\mathcal{E} \mathcal{L} \mathcal{H} \mathcal{I} \mathcal{F}_{\perp}$-to-

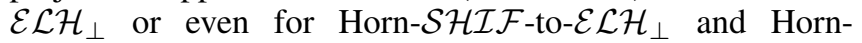
$\mathcal{S} \mathcal{R} \mathcal{I} \mathcal{F}$-to- $\mathcal{E} \mathcal{L} \mathcal{R}_{\perp}$. It would also be interesting to further extend the expressive power of both the source and target DLs. For example, nominals and range restrictions could be added even without compromising tractability of the latter [Baader et al., 2005]. We remark that Theorem 4 can be adapted to the extension $\mathcal{E} \mathcal{L} \mathcal{R}_{\perp}^{d r}$ of $\mathcal{E} \mathcal{L} \mathcal{R}_{\perp}$ with range restrictions as the target DL by additionally including in $\mathcal{O}_{T}$ the range restriction $\operatorname{ran}(r) \sqsubseteq \exists \hat{r}$. $\top$ for every role name $r$ in $\mathcal{O}_{S}$. Once more, inverse closedness pays off here as a corresponding extension of Theorem 3 appears to be more challenging.

There are many other relevant approximation cases that we did not touch upon, including the approximation of non-Horn DLs such as $\mathcal{A L C}, \mathcal{S H I} \mathcal{Q}$, and $\mathcal{S R O I \mathcal { Q }}$ in (tractable and intractable) Horn DLs. It would further be of interest to understand how approximations can be better tailored towards relevant applications, for example in the spirit of choosing a set $\Gamma$ of relevant concepts as discussed in Section 3.

\section{Acknowledgements}

Bötcher and Lutz were supported by DFG CRC 1320 Ease. 


\section{References}

[Baader et al., 2005] Franz Baader, Sebastian Brandt, and Carsten Lutz. Pushing the $\mathcal{E} \mathcal{L}$ envelope. In Proc. of $I J$ CAI, pages 364-369. Morgan Kaufmann, 2005.

[Baader et al., 2008] Franz Baader, Carsten Lutz, and Sebastian Brandt. Pushing the $\mathcal{E} \mathcal{L}$ envelope further. In Proc. of OWLED, volume 496 of CEUR Workshop Proceedings. CEUR-WS.org, 2008.

[Baader et al., 2017] Franz Baader, Ian Horrocks, Carsten Lutz, and Ulrike Sattler. An Introduction to Description Logic. Cambridge University Press, 2017.

[Beklemishev, 2014] Lev D. Beklemishev. Positive provability logic for uniform reflection principles. Ann. Pure Appl. Logic, 165(1):82-105, 2014.

[Bienvenu et al., 2016] Meghyn Bienvenu, Peter Hansen, Carsten Lutz, and Frank Wolter. First order-rewritability and containment of conjunctive queries in Horn description logics. In Proc. of IJCAI, pages 965-971. AAAI Press, 2016.

[Blackburn et al., 2001] Patrick Blackburn, Maarten de Rijke, and Yde Venema. Modal Logic. Cambridge University Press, 2001.

[Botoeva et al., 2010] Elena Botoeva, Diego Calvanese, and Mariano Rodriguez-Muro. Expressive approximations in DL-Lite ontologies. In Proc. of AIMSA, pages 21-31. Springer, 2010.

[Brandt et al., 2002] Sebastian Brandt, Rralf Küsters, and Anni-Yasmin Turhan. Approximation and difference in description logics. In Proc. of KR, pages 203-214. Morgan Kaufman, 2002.

[Carral et al., 2014] David Carral, Cristina Feier, Bernardo Cuenca Grau, Pascal Hitzler, and Ian Horrocks. $\mathcal{E} \mathcal{L}$-ifying ontologies. In Proc. of IJCAR, pages 464-479, 2014.

[Darwiche and Marquis, 2002] Adnan Darwiche and Pierre Marquis. A knowledge compilation map. J. Artif. Intell. Res., 17(1):229-264, 2002.

[Goldblatt, 1989] R. Goldblatt. Varieties of complex algebras. Ann. Pure Appl. Logic, 44(3):173-242, 1989.

[Groot et al., 2005] Perry Groot, Heiner Stuckenschmidt, and Holger Wache. Approximating description logic classification for semantic web reasoning. In Proc. of ESWC, pages 318-332. Springer, 2005.

[Jackson, 2004] Marcel Jackson. Semilattices with closure. Algebra Universalis, 52(1):1-37, 2004.

[Kikot et al., 2017] Stanislav Kikot, Ágnes Kurucz, Yoshihito Tanaka, Frank Wolter, and Michael Zakharyaschev. Kripke completeness of strictly positive modal logics over meet-semilattices with operators. CoRR, abs/1708.03403, 2017.

[Konev et al., 2016] Boris Konev, Carsten Lutz, Frank Wolter, and Michael Zakharyaschev. Conservative rewritability of description logic TBoxes. In Proc. of IJCAI, pages 1153-1159. AAAI Press, 2016.
[Konev et al., 2018] Boris Konev, Carsten Lutz, Ana Ozaki, and Frank Wolter. Exact learning of lightweight description logic ontologies. J. Mach. Learn. Res., 18(201):1-63, 2018.

[Kontchakov and Zakharyaschev, 2014] Roman Kontchakov and Michael Zakharyaschev. An introduction to description logics and query rewriting. In Proc. of $R W$, volume 8714 of LNCS, pages 195-244. Springer, 2014.

[Krötzsch et al., 2013] Markus Krötzsch, Sebastian Rudolph, and Pascal Hitzler. Complexities of Horn description logics. ACM Trans. Comput. Log., 14(1):2:12:36, 2013.

[Lutz and Wolter, 2010] Carsten Lutz and Frank Wolter. Deciding inseparability and conservative extensions in the description logic $\mathcal{E} \mathcal{L}$. J. Symb. Comput., 45(2):194-228, 2010 .

[Lutz et al., 2011] Carsten Lutz, Robert Piro, and Frank Wolter. Description logic TBoxes: Model-theoretic characterizations and rewritability. In Proc. of IJCAI, pages 983-988. IJCAI/AAAI, 2011.

[Lutz et al., 2012] Carsten Lutz, Inanç Seylan, and Frank Wolter. An automata-theoretic approach to uniform interpolation and approximation in the description logic $\mathcal{E} \mathcal{L}$. In Proc. of KR, pages 286-296. AAAI Press, 2012.

[Pan and Thomas, 2007] Jeff Z. Pan and Edward Thomas. Approximating $O W L-D L$ ontologies. In Proc. of $A A A I$, pages 1434-1439. AAAI Press, 2007.

[Ren et al., 2010] Yuan Ren, Jeff Z. Pan, and Yuting Zhao. Soundness preserving approximation for TBox reasoning. In Proc. of AAAI, pages 351-356. AAAI Press, 2010.

[Schaerf and Cadoli, 1995] Marco Schaerf and Marco Cadoli. Tractable reasoning via approximation. Artificial Intelligence, 74(2):249 - 310, 1995.

[Selman and Kautz, 1996] Bart Selman and Henry A. Kautz. Knowledge compilation and theory approximation. $J$. ACM, 43(2):193-224, 1996.

[Sofronie-Stokkermans, 2013] Viorica SofronieStokkermans. Locality and applications to subsumption testing in $\mathcal{E} \mathcal{L}$ and some of its extensions. Sci. Ann. Comp. Sci., 23(2):251-284, 2013.

[Sofronie-Stokkermans, 2017] Viorica SofronieStokkermans. Representation theorems and locality for subsumption testing and interpolation in the description logics $\mathcal{E} \mathcal{L}$ and $\mathcal{E} \mathcal{L}^{+}$and their extensions with $n$-ary roles and numerical domains. Fundam. Inform., 156(3-4):361-411, 2017.

[van Benthem, 1984] Johan van Benthem. Correspondence theory. In D. Gabbay and F. Guenthner, editors, Handbook of Philosophical Logic, volume 2, pages 167-247. Reidel, Dordrecht, 1984. 